\title{
Functional Quantum Nodes for Entanglement Distribution over Scalable Quantum Networks
}

\author{
Chin-Wen Chou, Julien Laurat, Hui Deng, Kyung Soo Choi, Hugues de Riedmatten*, Daniel Felinto ${ }^{\dagger}$, H. Jeff Kimble
}

Norman Bridge Laboratory of Physics 12-33, California Institute of Technology, Pasadena, California 91125, USA

(Dated: February 1, 2008)

\begin{abstract}
We demonstrate entanglement distribution between two remote quantum nodes located 3 meters apart [1]. This distribution involves the asynchronous preparation of two pairs of atomic memories and the coherent mapping of stored atomic states into light fields in an effective state of near maximum polarization entanglement. Entanglement is verified by way of the measured violation of a Bell inequality, and can be used for communication protocols such as quantum cryptography. The demonstrated quantum nodes and channels can be used as segments of a quantum repeater, providing an essential tool for robust long-distance quantum communication.
\end{abstract}

In quantum information science 2], distribution of entanglement over quantum networks is a critical requirement, including for metrology [3], quantum computation [4, 5] and communication [4, 6]. Quantum networks are composed of quantum nodes for processing and storing quantum states, and quantum channels that link the nodes. Significant advances have been made with diverse systems towards the realization of such networks, including ions [7], single trapped atoms in free space [8, 9] and in cavities [10], and atomic ensembles in the regime of continuous variables [11].

An approach of particular importance has been the seminal work of Duan, Lukin, Cirac, and Zoller (DLCZ) for the realization of quantum networks based upon entanglement between single photons and collective excitations in atomic ensembles [12]. Critical experimental capabilities have been achieved, beginning with the generation of nonclassical fields [13, 14] with controlled waveforms [15] and extending to the creation and retrieval of single collective excitations [16, 17, 18] with high efficiency [19, 20]. Heralded entanglement with quantum memory, which is the cornerstone of networks with efficient scaling, was achieved between two ensembles 21]. More recently, conditional control of the quantum states of a single ensemble [22, 23, 24] and of two distant ensembles 25] has also been implemented, as are likewise required for the scalability of quantum networks based upon probabilistic protocols.

Our interest is to develop the physical resources that enable quantum repeaters [6], thereby allowing entanglement-based quantum communication tasks over quantum networks on distance scales much larger than set by the attenuation length of optical fibers, including quantum cryptography [26]. For this purpose, heralded number state entanglement [21] between two remote atomic ensembles is not directly applicable. In-

\footnotetext{
*Current address: Group of Applied Physics, University of Geneva, Geneva, Switzerland

${ }^{\dagger}$ Current address: Departamento de Física, Universidade Federal de Pernambuco, Recife-PE, 50670-901, Brazil
}

stead, DLCZ proposed to use pairs of ensembles $\left(U_{i}, D_{i}\right)$ at each quantum node $i$, with the sets of ensembles $\left\{U_{i}\right\},\left\{D_{i}\right\}$ separately linked in parallel chains across the network [12]. Relative to the state of the art in Ref. [21], the DLCZ protocol requires the capability for the independent control of pairs of entangled ensembles between two nodes.

In our experiment, we have created, addressed, and controlled pairs of atomic ensembles at each of two quantum nodes, thereby demonstrating entanglement distribution in a form suitable both for quantum network architectures and for entanglement-based quantum communication schemes. Specifically, two pairs of remote ensembles at two nodes are each prepared in an entangled state [21], in a heralded and asynchronous fashion [25], thanks to the conditional control of the quantum memories. After a signal heralding that the two chains are prepared in the desired state, the states of the ensembles are coherently transferred to propagating fields locally at the two nodes. The fields are arranged such that they effectively contain two photons, one at each node, whose polarizations are entangled. The entanglement between the two nodes is verified by the violation of a Bell inequality. The effective polarization entangled state, created with favorable scaling behavior, is thereby compatible with entanglement-based quantum communication protocols [12].

The architecture for our experiment is shown in Figure 1. Each quantum node, $L$ (left) and $R$ (right), consists of two atomic ensembles, $U$ (up) and $D$ (down), or four ensembles altogether, namely $(L U, L D)$ and $(R U$, $R D)$, respectively. Each pair is first prepared in an entangled state, where one excitation is shared coherently, by using a pair of coherent weak write pulses to induce spontaneous Raman transitions $|g\rangle \rightarrow|e\rangle \rightarrow|s\rangle$, as illustrated in the inset in Fig. 1. The Raman fields $\left(1_{L U}, 1_{R U}\right)$ from $(L U, R U)$ are combined at the 50-50 beamsplitter $B S_{U}$, with the resulting fields directed to single-photon detectors. A photoelectric detection event in either detector heralds that the two ensembles are prepared. The remote pair of $D$ ensembles, $(L D, R D)$, is prepared in an analogous fashion. 


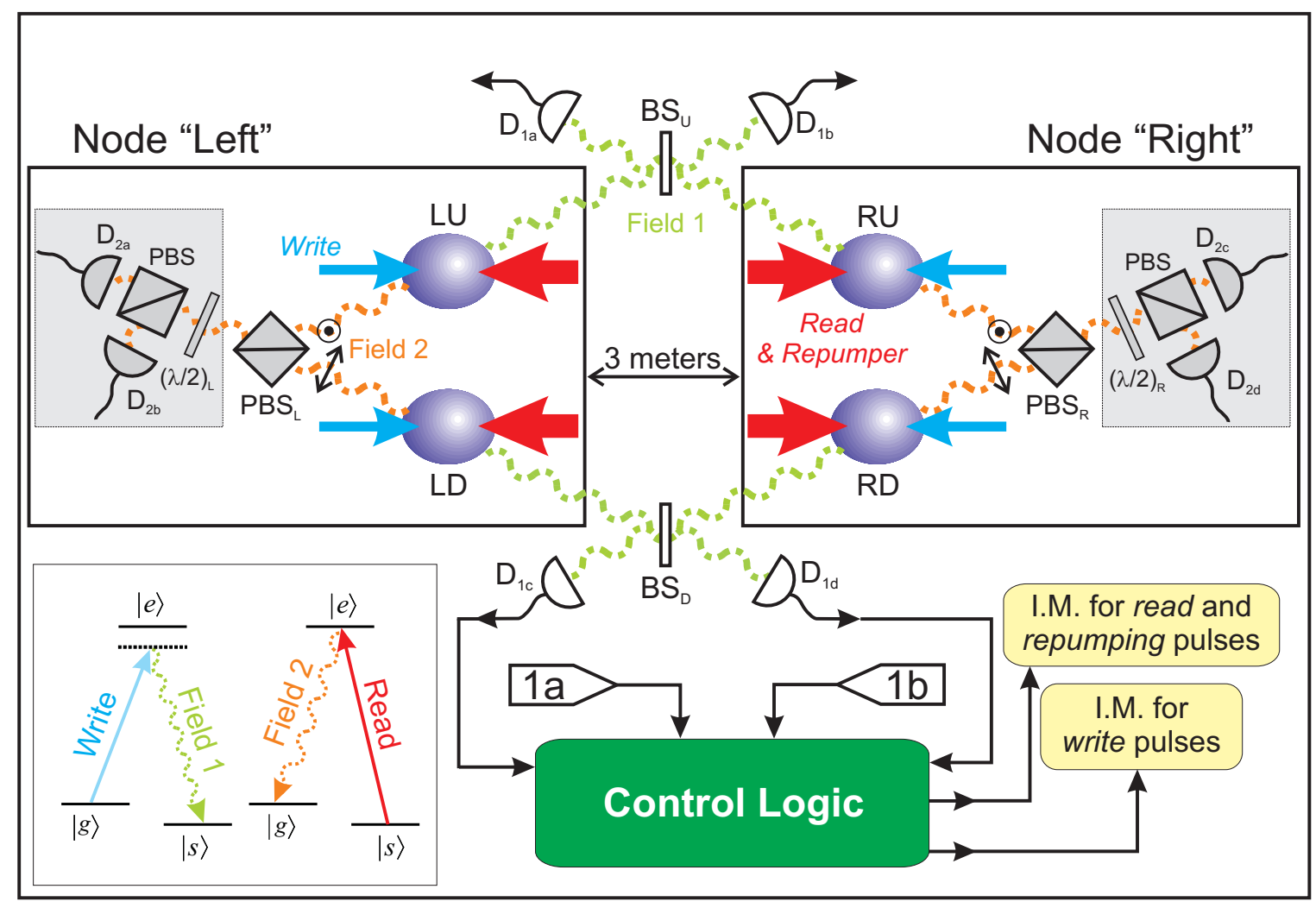

FIG. 1: Setup for distributing entanglement between two quantum nodes $(L, R)$ separated by 3 meters. The inset shows the relevant atomic levels for the $6 S_{1 / 2} \rightarrow 6 P_{3 / 2}$ transition in atomic Cesium, as well as the associated light fields. The ensembles are initially prepared in $|g\rangle$. Weak write pulses then induce spontaneous Raman transitions $|g\rangle \rightarrow|e\rangle \rightarrow|s\rangle$, resulting in the emission of anti-Stokes fields (fields 1) near the $|e\rangle \rightarrow|s\rangle$ transition along with the storage of collective excitations in the form of spin-flips shared among the atoms 12]. With this setup, a photo-detection event at either detector $D_{1 a}$ or $D_{1 b}\left(D_{1 c}\right.$ or $\left.D_{1 d}\right)$ heralds entanglement between the collective excitation in $L U$ and $R U$ ( $L D$ and $\left.R D\right)$ [21] (see text). $B S_{U}$ and $B S_{D}$ are implemented using two orthogonal polarizations in one fiber beam splitter, yielding excellent relative path stability. A heralding detection event triggers the control logic to gate off the light pulses going to the corresponding ensemble pair $(U$ or $D)$ by controlling the intensity modulators $(I M)$. The atomic state is thus stored while waiting for the second ensemble pair to be prepared. After both pairs of ensembles $U, D$ are entangled, the control logic releases strong read pulses to map the states of the atoms to Stokes fields 2 via $|s\rangle \rightarrow|e\rangle \rightarrow|g\rangle$. Fields $2_{L U}$ and $2_{L D}\left(2_{R U}\right.$ and $\left.2_{R D}\right)$ are combined with orthogonal polarizations on the polarizing beam splitter $P B S_{L}\left(P B S_{R}\right)$ to yield field $2_{L}\left(2_{R}\right)$. If only coincidences between fields $2_{L}$ and $2_{R}$ are registered, the state is effectively equivalent to a polarization maximally entangled state. $(\lambda / 2)_{L, R}:$ rotatable half-wave plates.

Conditioned upon the preparation of both ensemble pairs $(L U, L D)$ and $(R U, R D)$, a set of read pulses are triggered to map the stored atomic excitations into propagating Stokes fields in well-defined spatial modes via $|s\rangle \rightarrow|e\rangle \rightarrow|g\rangle$ by way of a collective enhancement
12] (see inset in Fig. 1). This generates a set of four fields denoted by $\left(2_{L U}, 2_{R U}\right)$ for ensembles $(L U, R U)$ and $\left(2_{L D}, 2_{R D}\right)$ for ensembles $(L D, R D)$. In the ideal case and neglecting higher-order terms, this mapping results in a quantum state for the fields 2 given by

$$
\left|\psi_{2_{L U}, 2_{R U}, 2_{L D}, 2_{R D}}\right\rangle=\frac{1}{2}\left(|0\rangle_{2_{L U}}|1\rangle_{2_{R U}} \pm e^{i \eta_{U}}|1\rangle_{2_{L U}}|0\rangle_{2_{R U}}\right)_{U} \otimes\left(|0\rangle_{2_{L D}}|1\rangle_{2_{R D}} \pm e^{i \eta_{D}}|1\rangle_{2_{L D}}|0\rangle_{2_{R D}}\right)_{D}
$$

Here, $|n\rangle_{x}$ is the $n$-photon state for mode $x$, where $x \in\left\{2_{L U}, 2_{R U}, 2_{L D}, 2_{R D}\right\}$, and $\eta_{U}\left(\eta_{D}\right)$ is the relative phase resulting from the writing and reading processes for the $U(D)$ pair of ensembles [21]. The \pm signs for the conditional states $U, D$ result from the unitarity of the transformation by the beamsplitters $\left(B S_{U}, B S_{D}\right)$. The 
extension of Eq. (11) to incorporate various nonidealities is given in the Appendix.
Apart from an overall phase, the state $\left|\psi_{2_{L U}, 2_{R U}, 2_{L D}, 2_{R D}}\right\rangle$ can be rewritten as follows: where $|v a c\rangle_{2_{i}}$ denotes $|0\rangle_{2_{i U}}|0\rangle_{2_{i D}}$. If only coincidences between both nodes $L, R$ are registered, the first two terms (i.e., with $e^{-i \eta_{D}}, e^{i \eta_{U}}$ ) do not contribute. Hence, as noted by DLCZ, excluding such cases leads to an effective density matrix equivalent to the one for a maximally entangled state of the form of the last term in Eq. (2). Significantly, the absolute phases $\eta_{U}$ and $\eta_{D}$ do not need to be independently stabilized. Only the relative phase $\eta=\eta_{U}-\eta_{D}$ must be kept constant, leading to $1 / 2$ unit of entanglement for two quantum bits (i.e., $1 / 2$ ebit).

The experimental demonstration of this architecture for implementing the DLCZ protocol relies critically on the ability to carry out efficient parallel preparation of the $(L U, R U)$ and $(L D, R D)$ ensemble pairs, as well as to stabilize the relative phase $\eta$. The first requirement is achieved by the use of real-time control, as described in Ref. 25] in a simpler case. As illustrated in Fig. 1, here we implement control logic that monitors the outputs of field 1 detectors. A detection event at either pair triggers electro-optic intensity modulators (IM) that gate off all laser pulses going to the corresponding pair of ensembles, thereby storing the associated state. Upon receipt of signals heralding that the two pairs of ensembles $(L U$, $R U)$ and $(L D, R D)$ have both been independently prepared, the control logic triggers the retrieval of the stored states by simultaneously sending a strong read pulse into each of the four ensembles. Relative to the case where no logic is implemented, a 19-fold enhancement is obtained in the probability to generate this overall state from the four ensembles (see Appendix).

The second requirement, for stability of the relative phase $\eta$, could be accomplished by active stabilization of each individual phase $\eta_{U}, \eta_{D}$, as in Ref. 21]. Instead of implementing this challenging technical task (which ultimately would have to be extended across longer chains of ensembles), our setup exploits the passive stability between two independent polarizations propagating in a single interferometer to prepare the two ensemble pairs [27]. No active phase stabilization is thus required. In practice, we find that the passive stability of our system is sufficient for operation overnight without adjustment. Additionally, we implement a procedure that deterministically sets the relative phase $\eta$ to zero.

We also extend the original DLCZ protocol by, as illustrated in Fig. 1, combining fields $\left(2_{L U}, 2_{L D}\right)$ and $\left(2_{R U}\right.$,
$\left.2_{R D}\right)$ with orthogonal polarizations on polarizing beam splitters $P B S_{L}$ and $P B S_{R}$ to yield fields $2_{L}$ and $2_{R}$, respectively. The polarization encoding opens the possibility of performing additional entanglement purification and thus superior scalability [28, 29]. In the ideal case, the resulting state is now effectively equivalent to a maximally entangled state for the polarization of two photons

$$
\left|\psi_{2_{L}, 2_{R}}^{ \pm}\right\rangle_{e f f} \propto\left|H_{2_{L}}\right\rangle\left|V_{2_{R}}\right\rangle \pm e^{i \eta}\left|V_{2_{L}}\right\rangle\left|H_{2_{R}}\right\rangle,
$$

where $|H\rangle(|V\rangle)$ stands for the state of a single photon with horizontal (vertical) polarization. The sign of the superposition in Eq. (3) is inherited from Eq. (1) and is determined by the particular pair of heralding signals recorded by $\left(D_{1 a}, D_{1 b}\right)$ and $\left(D_{1 c}, D_{1 d}\right)$. The entanglement in the polarization basis is well-suited for entanglementbased quantum cryptography [12, 26], including security verification by way of the violation of a Bell inequality, as well as for quantum teleportation [12].

As a first step to investigate the joint states of the atomic ensembles, we record photoelectric counting events for the ensemble pairs $(L U, R U)$ and $(L D, R D)$ by setting the angles for the half-wave plates $\left(\frac{\lambda}{2}\right)_{L, R}$ shown in Fig. 1 to $0^{\circ}$ such that photons reaching detectors $D_{2 b}$ and $D_{2 d}\left(D_{2 a}\right.$ and $\left.D_{2 c}\right)$ come only from the ensemble pair $U(D)$. Conditioned upon detection events at $D_{1 a}$ or $D_{1 b}\left(D_{1 c}\right.$ or $\left.D_{1 d}\right)$, we estimate the probability that each ensemble pair $U, D$ contains only a single, shared excitation as compared to the probability for two excitations by way of the associated photoelectric statistics. In quantitative terms, we determine the ratio [21]

$$
h_{X}^{(2)} \equiv \frac{p_{X, 11}}{p_{X, 10} p_{X, 01}},
$$

where $p_{X, m n}$ are the probabilities to register $m$ photodetection events in mode $2_{L X}$ and $n$ events in mode $2_{R X}$ $(X=\{U, D\})$ conditioned on a detection event at $D_{1}$. A necessary condition for the two ensembles $(L X, R X)$ to be entangled is that $h_{X}^{(2)}<1$, where $h_{X}^{(2)}=1$ corresponds to the case of independent (unentangled) coherent states for the two fields [21]. Fig. 2 shows the measured $h_{X}^{(2)}$ versus the duration $\tau_{M}$ that the state is stored before retrieval. For both $U$ and $D$ pairs, $h^{(2)}$ remains well 


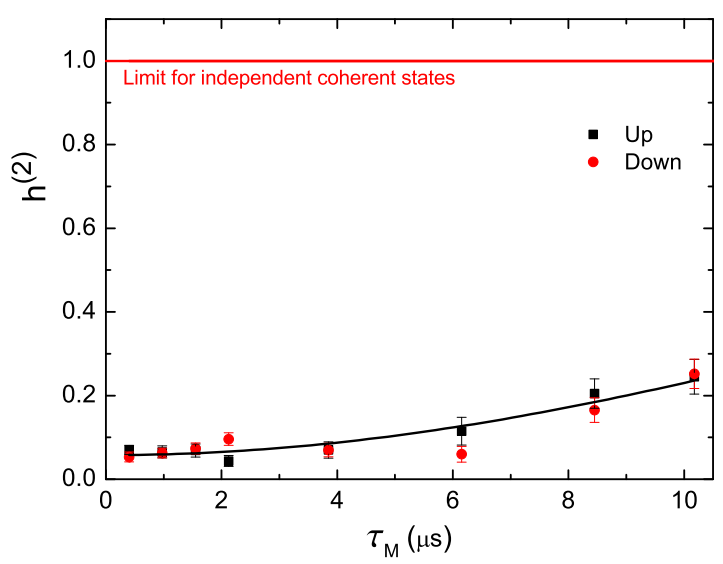

FIG. 2: Suppression $h^{(2)}$ of the probabilities for each ensemble to emit two photons compared to the product of the probabilities that only one photon is emitted, as a function of the duration $\tau_{M}$ that the state is stored before retrieval. The solid line gives a fit for the $U$ pair. Error bars indicate statistical errors.

below unity for storage times $\tau_{M} \lesssim 10 \mu \mathrm{s}$. For the $U$ pair, the solid line in Fig. 2 provides a fit by the simple expression $h^{(2)}=1-A \exp \left(-\left(\tau_{M} / \tau\right)^{2}\right)$. The fit gives $A=0.94 \pm 0.01$ and $\tau=22 \pm 2 \mu s$, providing an estimate of a coherence time for our system. A principal cause for decoherence is an inhomogeneous broadening of the ground state levels by residual magnetic fields 30]. The characterization of the time dependence of $h^{(2)}$ constitutes an important benchmark of our system, as will be clarified shortly (also see Appendix).

We next measure the correlation function $E\left(\theta_{L}, \theta_{R}\right)$ defined by

$$
E\left(\theta_{L}, \theta_{R}\right)=\frac{C_{a c}+C_{b d}-C_{a d}-C_{b c}}{C_{a c}+C_{b d}+C_{a d}+C_{b c}} .
$$

Here, $C_{j k}$ gives the rates of coincidences between detectors $D_{2 j}$ and $D_{2 k}$ for fields 2 , where $j, k \in\{a, b, c, d\}$, conditioned upon heralding events at detectors $D_{1 a}, D_{1 b}$ and $D_{1 c}, D_{1 d}$ from fields 1 . The angles of the two half-wave plates $\left(\frac{\lambda}{2}\right)_{L, R}$ are set at $\theta_{L} / 2$ and $\theta_{R} / 2$, respectively. As stated before, the capability to store the state heralded in one pair of ensembles and then to wait for the other pair to be prepared significantly improves the various coincidence rates $C_{j k}$ by a factor that increases with the duration $\tau_{M}$ that a state can be preserved [25] (also see Appendix).

Fig. 3 displays the correlation function $E$ as a function of $\theta_{R}$, with $\theta_{L}=0^{\circ}$ in $\mathrm{A}$ and $45^{\circ}$ in B . Relative to Fig. 2, these data are taken with increased excitation probability (higher write power) to validate the phase stability of the system, which is evidently good. Moreover, these four-fold coincidence fringes in Fig. 3A provide a further verification that predominantly one excitation is shared between a pair of ensembles. The analysis provided in the Appendix with the measured cumulative $h^{(2)}$ parameter for this set of data, $h^{(2)}=0.12 \pm 0.02$, predicts a visibility of $V=78 \pm 3 \%$ in good agreement with the experimentally determined $V \cong 75 \%$. Finally, the fact that one of the fringes is inverted with respect to the other in Fig. 3B corresponds to the two possible signs in Eq. (3). As for $\theta_{L}=45^{\circ}$ the measurement is sensitive to the square of the overlap $\xi$ of photon wavepackets for fields $2_{U, D}$, we may infer $\xi_{U, D} \simeq 0.85$ from the reduced fringe visibility $(V \cong 55 \%)$ in Fig. $3 \mathrm{~B}$ relative to $\mathrm{A}$, if all the reduction is attributed to a nonideal overlap. An independent experiment for two-photon interference in this setup has shown an overlap $\xi \simeq 0.90$ (see Appendix), which confirms that the reduction can be principally attributed to the non-ideal overlap. Other possible causes include imperfect phase alignment $\eta \neq 0$ and imbalance of the effective state coefficients (see Appendix).

With the measurements from Figs. 2, 3 in hand, we verify entanglement unambiguously by way of the violation of a Bell inequality [31]. For this purpose, we choose the canonical values, $\theta_{L}=\left\{0^{\circ}, 45^{\circ}\right\}$ and $\theta_{R}=$ $\left\{22.5^{\circ},-22.5^{\circ}\right\}$, and construct the $\mathrm{CHSH}$ parameters

$$
\begin{aligned}
S_{+} \equiv & \mid E\left(0^{\circ}, 22.5^{\circ}\right)+E\left(0^{\circ},-22.5^{\circ}\right) \\
& +E\left(45^{\circ},-22.5^{\circ}\right)-E\left(45^{\circ}, 22.5^{\circ}\right) \mid \\
S_{-} \equiv & \mid E\left(0^{\circ}, 22.5^{\circ}\right)+E\left(0^{\circ},-22.5^{\circ}\right) \\
& +E\left(45^{\circ}, 22.5^{\circ}\right)-E\left(45^{\circ},-22.5^{\circ}\right) \mid
\end{aligned}
$$
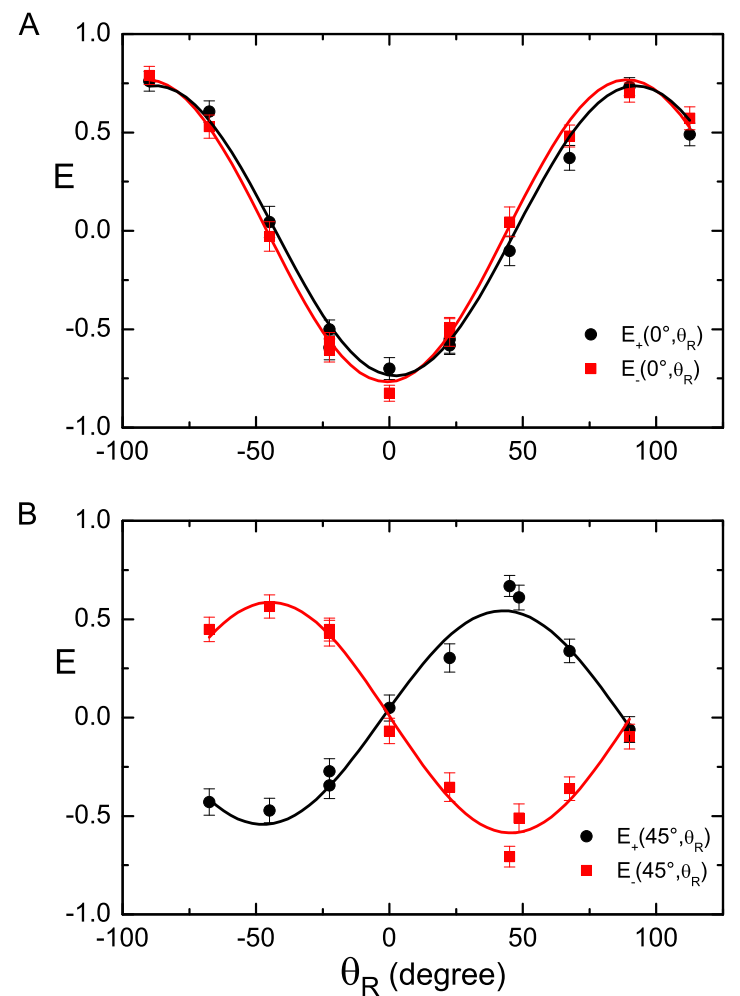

FIG. 3: Measured correlation function $E\left(\theta_{L}, \theta_{R}\right)$ as a function of $\theta_{R}$ with $\theta_{L}$ fixed at $\mathrm{A}: 0^{\circ}$ and $\mathrm{B}: 45^{\circ}$. The excitation probabilities for the ensembles are increased by $\sim 1.5$ times relative to Fig. 2, with each point taken for 30 minutes at typically 400/hour coincidence rate for each fringe. Error bars indicate statistical errors. 

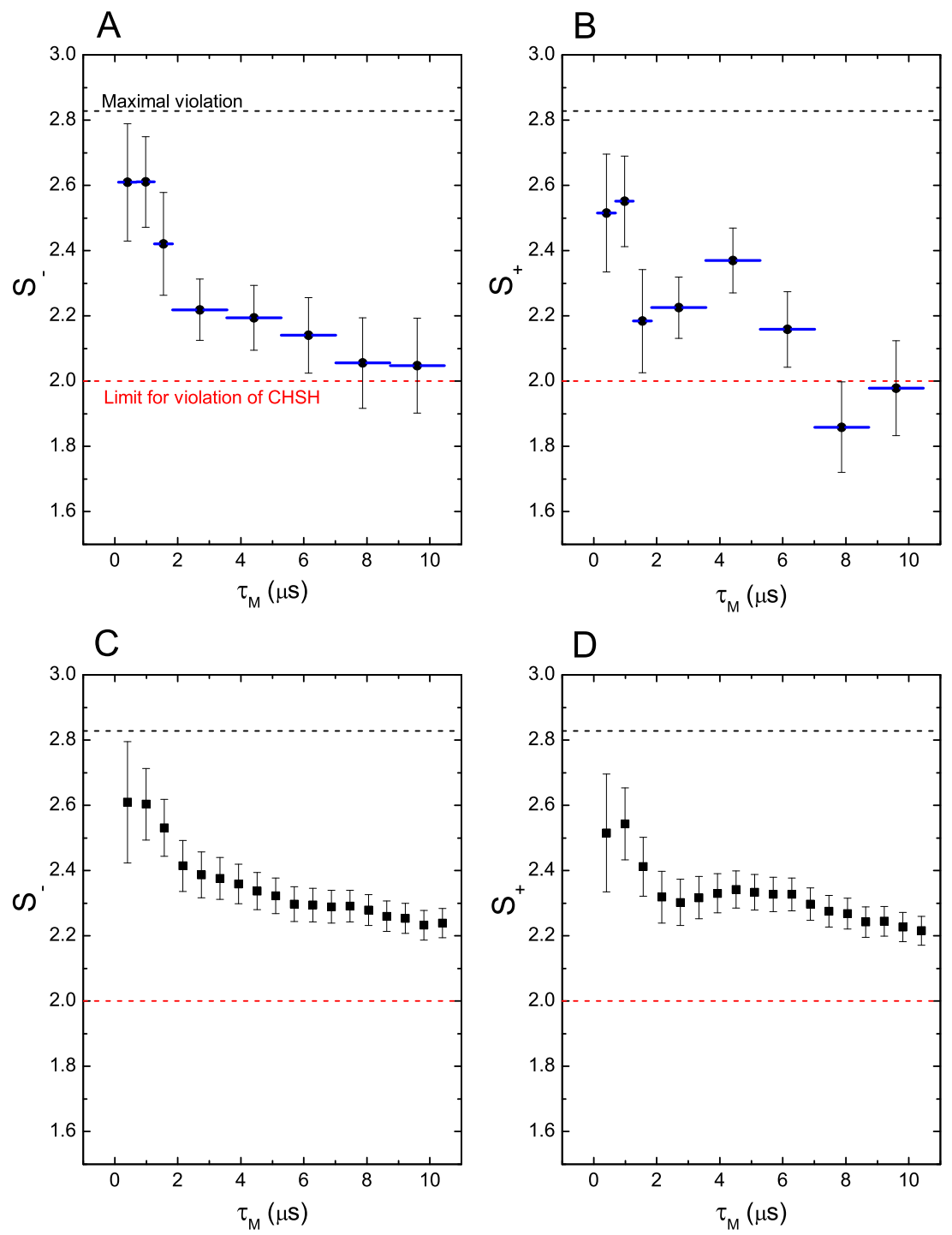

FIG. 4: Measured CHSH parameters $S_{ \pm}$, for the two possible effective states in Eq. (3), as functions of duration $\tau_{M}$ for which the first ensemble pair holds the prepared state. The excitation probabilities are kept low for high correlation (as in Fig. 2). A and B: binned data. The horizontal thick lines indicate the size of the bins used. C and D: cumulative data. The coincidence rate for these measurements is about 150/hour for each effective state. Error bars indicate statistical errors.

for the two effective states $\left|\psi_{2_{L}, 2_{R}}^{ \pm}\right\rangle_{\text {eff }}$ in Eq. (3). For local, realistic hidden variable theories, $S_{ \pm} \leq 2[31]$. Fig. 4 shows the CHSH parameters $S_{ \pm}$as functions of the duration $\tau_{M}$ up to which one pair of ensembles holds the prepared state, in the excitation regime of Fig. 2. As shown in the Appendix, the requirements for minimization of higher order terms are much more stringent in this experiment with four ensembles than with simpler configurations 22].

Panels A and B in Fig. 4 give the results for our measurements of $S_{ \pm}$with binned data. Each point corresponds to the violation obtained for states generated at $\tau_{M} \pm \Delta \tau_{M} / 2\left(\Delta \tau_{M}\right.$ marked by the thick horizontal lines in Fig. 4). Strong violations are obtained for short memory times, with for instance $S_{+}=2.55 \pm 0.14>2$ and $S_{-}=2.61 \pm 0.13>2$ for the second bin, demonstrat- ing the presence of entanglement between fields $2_{L}$ and $2_{R}$. Therefore, these fields can be exploited to perform entanglement-based quantum communication protocols, such as quantum key distribution with, at minimum, security against individual attacks [12, 32].

As it can be seen in Fig. 4, the violation decreases with increasing $\tau_{M}$. The decay is largely due to the timevarying behavior of $h^{(2)}$ (Fig. 2 and Appendix). In addition to this decay, the $S_{+}$parameter exhibits modulation with $\tau_{M}$. We have explored different models for the time dependence of the CHSH parameters, but thus far have found no satisfactory agreement between model calculations and measurements. Nevertheless, the density matrix for the ensemble over the full memory time is potentially useful for tasks such as entanglement connection, as shown by panels $\mathrm{C}$ and $\mathrm{D}$ where cumulative data are 
given. Each point at memory time $\tau_{M}$ gives the violation obtained by taking into account all the states generated from 0 to $\tau_{M}$. Overall significant violations are obtained, namely $S_{+}=2.21 \pm 0.04>2$ and $S_{-}=2.24 \pm 0.04>2$ at $\tau_{M} \sim 10 \mu \mathrm{s}$.

In our experiment, we are able to generate excitationnumber entangled states between remote locations, which are well suited for scaling purposes, and, with real-time control, to operate them as if they were effectively polarization entangled states, which are appropriate for quantum communication applications, such as quantum cryptography. Measurements of the suppression $h^{(2)}$ of twoexcitation components versus storage time demonstrates explicitly the major source that causes the extracted polarization entanglement to decay, emphasizing the critical role of multi-excitation events in the experiments aiming for a scalable quantum network. The present scheme, which constitutes a functional segment of a quantum repeater in terms of quantum state encoding and channel control, allows the distribution of entanglement between two quantum nodes. But the extension of our work to longer chains involving many segments becomes more complicated, and out of reach for any current system. For long-distance communication, the first quantity to improve is the coherence time of the memory. Better cancellation of the residual magnetic fields and switching to new trap schemes should improve this parameter to $\sim 0.1 \mathrm{sec}$ by employing an optical trap [30], thereby increasing the rate of preparing the ensembles in the state of Eq. (11) to $\sim 100 \mathrm{~Hz}$. The second challenge which would immediately appear in an extended chain would be the increase of the multi-excitation probability with the connection stages. Recently, Ref. 28] has theoretically demonstrated the prevention of such growth in a similar setup, but its full scalability still requires very high retrieval and detection efficiency, and photon-number resolving detectors. These two points clearly show that the quest of scalable quantum networks is still a theoretical and experimental challenge. The availability of our first functional segment opens the way for fruitful investigations.

\section{APPENDIX A: EXPERIMENTAL DETAILS}

Ensembles $(L U, L D)$ are pencil-shaped groups of cold Cesium atoms in a magneto-optical trap (MOT) while ensembles $(R U, R D)$ are in another MOT, 3 meters away. $\quad\{|g\rangle,|s\rangle,|e\rangle\}$ correspond to the hyperfine levels $\left\{\left|6 S_{1 / 2}, F=4\right\rangle,\left|6 S_{1 / 2}, F=3\right\rangle,\left|6 P_{3 / 2}, F^{\prime}=4\right\rangle\right\}$, respectively. In each MOT, the ensembles $U, D$ are separated by $1 \mathrm{~mm}$ by way of birefringent beam displacers [27]. The MOT is formed at a repetition rate of $40 \mathrm{~Hz}$. In each cycle, the MOT is loaded for $18 \mathrm{~ms}$, after which the magnetic field is quickly switched off. The trapping beams are turned off $3 \mathrm{~ms}$ after the magnetic field, while the repumping beam stays on for another $100 \mu$ s before being switched off in order to prepare the atoms in the $F=4$ ground state $|g\rangle .3 .4 \mathrm{~ms}$ after the magnetic field is turned off, trials of the protocol (each consisting of successive write, read, and repumping pulses) are repeated with 575 ns period for $3.4 \mathrm{~ms}$. In each trial, the write pulse is $\approx 30 \mathrm{~ns}$ in duration and $10 \mathrm{MHz}$ red-detuned from the $|g\rangle \rightarrow|e\rangle$ transition. The read pulse and the repumping pulse are both derived from the read beam (resonant with the $|s\rangle \rightarrow|e\rangle$ transition) with 30 ns and 75 ns duration, respectively. The read pulse is closely followed by the repumping pulse. The read pulse is delayed $\approx 400 \mathrm{~ns}$ after the write pulse, leaving time for the control logic to gate it off, along with the subsequent pulses. Independent phase stability measurements show that the phase $\eta$ drifts in a negligible way, $(\pi / 30)$ over $500 \mu s$ corresponding to 870 trials. Some other parameters of the experiments are calibrated and listed in table [.

\section{APPENDIX B: FRINGE VISIBILITY AS A FUNCTION OF $h^{(2)}$}

Let us consider that the two pairs of ensembles, $U$ and $D$, have been prepared by heralded detections at $D_{1 a}, D_{1 b}$ and $D_{1 c}, D_{1 d}$. Denote by $p_{10}, p_{01}$, and $p_{11}$ the probability $p_{i j}$ to register $i$ photodetection events in field $2_{L U}$ and $j$ in field $2_{R U}$ after firing the read pulses. We will assume, for simplicity, the various $p_{i j}$ are the same for both pairs of ensembles. For each of them, the suppression of the two-photon events relative to the square of the probability for single-photon events is characterized by the parameter $h^{(2)}[21]$ :

$$
h^{(2)}=\frac{p_{11}}{p_{10} p_{01}} .
$$

We next relate $h^{(2)}$ to the maximal $C_{\max }$ and minimal $C_{m i n}$ coincidence probabilities between various output ports of the detection polarizing beamsplitters $(P B S)$ for the left and right nodes at detectors $D_{2 a}, D_{2 b}$ and $D_{2 c}, D_{2 d}$ (see Fig. 1 of the main text). Consider, for example, the transmitted ports of the $P B S$ at the $L, R$ detectors for the case that the left node has the half-wave plate $\left(\frac{\lambda}{2}\right)_{L}$ set to $0^{\circ}$. In this case, fields $2_{L U}$ and $2_{L D}$ are detected independently, with field $2_{L D}$ transmitted at the $P B S$. On one hand, $C_{\max }$ is obtained for crossed polarizers (i.e., $\left(\frac{\lambda}{2}\right)_{R}$ set to $45^{\circ}$ at the right node, with then field $2_{R U}$ transmitted) and is given to lowest order by:

$$
C_{\max }=p_{10} p_{01}
$$

This term corresponds to the case where only a single excitation is distributed in each pair, and each retrieved photon is detected from a transmitted port on each side $L, R$.

On the other hand, the minimum coincidence probability $C_{\min }$ is obtained for parallel polarizers (i.e., $\left(\frac{\lambda}{2}\right)_{R}=$ $0^{\circ}$ at the right node, with then field $2_{R D}$ transmitted) 
TABLE I: Noise and Efficiencies

\begin{tabular}{|c|c|c|}
\hline & $\mathrm{U}$ & $\mathrm{D}$ \\
\hline Field 1 dark count rate & $\sim 10 \mathrm{~Hz}$ & $\sim 10 \mathrm{~Hz}$ \\
\hline Field 2 dark count rate & $\sim 100 \mathrm{~Hz}$ & $\sim 100 \mathrm{~Hz}$ \\
\hline Overall retrieval efficiency $p_{c}$ & $6.4 \% \pm 0.5 \%$ & $8.0 \% \pm 0.5 \%$ \\
\hline Field 2 propagation loss & $68 \pm 5 \%$ & $68 \pm 5 \%$ \\
\hline Field 2 photon detection efficiency & $50 \pm 5 \%$ & $50 \pm 5 \%$ \\
\hline
\end{tabular}

and can be written as:

$$
C_{\min }=p_{11}
$$

This term corresponds to coincidences due to photons coming from the same pair of ensembles. The smaller is the excitation probability, the smaller is this background term.

Taking Eqs. (B2) and (B3) into account, we find that the visibility for the number of coincidences as a function of the right polarizer angle (i.e., the angle for $\left.\left(\frac{\lambda}{2}\right)_{R}\right)$ is given by:

$$
V=\frac{C_{\max }-C_{\min }}{C_{\max }+C_{\min }}=\frac{1-h^{(2)}}{1+h^{(2)}} .
$$

Assuming that the visibility is the same in each basis, we then find a CHSH parameter $S$ equal to [33]:

$$
S=2 \sqrt{2} V=2 \sqrt{2} \frac{1-h^{(2)}}{1+h^{(2)}} .
$$

A minimal value $h_{\min }^{(2)}=0.17$ is thus required to violate the CHSH inequality $S<2$ in the absence of any imperfections except the intrinsic two-photon component. This value underlines that this experiment is much more stringent in terms of minimization of high-order terms than previously reported setups. For example, in Ref. [22], where entanglement between a photon and a stored excitation is reported, a value of $h^{(2)}$ equal to 0.68 was sufficient to violate the inequality. The dramatic improvement reported recently by different groups for the quality of the photon pairs emitted by an atomic ensemble was thus an enabling step for the practical realization of such a more elaborate procedure involving a total of 4 ensembles reported in the main text.

\section{APPENDIX C: TWO-PHOTON INTERFERENCE AND INFERRED OVERLAPS}

For a non-perfect overlap $\xi$ of the field-2 photon wavepackets, the visibility of the fringes in the $45^{\circ}$ basis is decreased by a factor $\xi^{2}$. This overlap can be determined by two-photon interference, which is implemented by mixing the fields $2_{U}$ and $2_{D}$ on each side (Right and Left) by rotating the half-wave plates $\left(\frac{\lambda}{2}\right)_{L},\left(\frac{\lambda}{2}\right)_{R}$ by $22.5^{\circ}$. If the single photon wavepackets are indistinguishable, no coincidences should be observed. However, the two-photon component can lead to coincidences, which reduce the visibility. Let us determine the expected visibility as a function of the two-photon component by way of a simple model.

Consider $P_{n}$ the probability of finding $n$ photons in field 2, and assume the various $P_{n}$ are the same for both ensembles involved. In the ideal case where all ensembles have the same properties, the two-photon suppression for each field 2 can also be characterized by the same $h^{(2)}$ parameter used before, which can be written here as:

$$
h^{(2)}=\frac{2 P_{2}}{P_{1}^{2}} \text {. }
$$

When the half-wave plates $\left(\frac{\lambda}{2}\right)_{L},\left(\frac{\lambda}{2}\right)_{R}$ are at $0^{\circ}$, the fields 2 are detected independently and the probability $p_{\max }$ to register coincidences is given by:

$$
p_{\max }=P_{1}^{2} \text {. }
$$

When the half-wave plates $\left(\frac{\lambda}{2}\right)_{L},\left(\frac{\lambda}{2}\right)_{R}$ are rotated to $22.5^{\circ}$, if the two fields overlap perfectly, the term with one photon in each input does not lead to coincidences. If we denote by $\xi$ the overlap, the probability $p_{\min }$ to have one photon in each output is then:

$$
p_{\text {min }}=\frac{\left(1-\xi^{2}\right)}{2} P_{1}^{2}+\frac{P_{2}}{2}+\frac{P_{2}}{2}=\left[1-\xi^{2}+h^{(2)}\right] \frac{P_{1}^{2}}{2} .
$$

From these two probabilities, we find that the visibility of the dip in coincidences can be written as:

$$
V_{d i p}=\frac{p_{\max }-p_{\min }}{p_{\max }}=\frac{1+\xi^{2}-h^{(2)}}{2} .
$$

In our case, the measured visibility $V_{d i p}$ is $85 \pm 2 \%$ for the left node and $89 \pm 2 \%$ for the right one. The measured average $h^{(2)}$ parameter for this set of data is $0.09 \pm 0.01$, which should lead in the case of perfect overlap to visibilities $V_{\text {model }}=95.5 \pm 0.5 \%$. From the measured visibilities and this simple model, we can then estimate the overlaps: $\xi=0.89 \pm 0.03$ for the left node and $\xi=0.93 \pm 0.03$ for the right node.

\section{APPENDIX D: DECOHERENCE TIME OF THE STORED EXCITATION}

Residual magnetic fields, which lead to inhomogeneous broadening of the ground states levels, is the major limiting factor of the coherence time $\tau_{c}$ of the stored excitation [22, 30]. Consequently, if we neglect dark counts, the 
conditional retrieval efficiency $p_{c}=p_{01}+p_{10}$ is expected to decay exponentially with the storage time $\tau_{M}$ :

$$
p_{c}=p_{c}^{0} \exp \left(-\frac{\tau_{M}}{\tau_{c}}\right) \text {. }
$$

Figure 5 shows an independent measurement of $p_{c}$ vs. $\tau_{M}$, with the $U$ and $D$ pairs separated. Fitting the data with Eq. (D1) gives, for the $\mathrm{U}$ and $\mathrm{D}$ pairs respectively, $p_{c}^{0}=7.0 \% \pm 0.1 \%$ and $8.7 \% \pm 0.2 \%$, and $\tau_{c}=9.1 \pm 0.6 \mu \mathrm{s}$ and $8.5 \pm 0.5 \mu \mathrm{s}$.

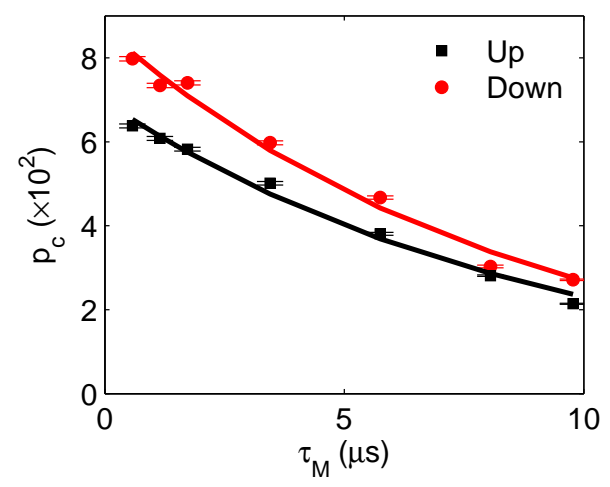

FIG. 5: Conditional probability $p_{c}$ of detecting one photon in a field 2 for the U (black squares) and D (red circles) pairs, as a function of the storage time $\tau_{M}$ of an excitation. The error bars indicate statistical errors. The solid lines are fits using Eq. (D1).

The decay of $p_{c}$ leads to a similar exponential decay of $C_{i j} . C_{i j}(i, j=a, b, c, d)$ are the coincidence count rates of two field 2 photons conditioned on two heralding field 1 photons defined before. Summing over all $C_{i j}$ used in calculating $S_{ \pm}$, we obtain the total coincidence count rates $C_{S \pm}$ for the measurement of the Bell parameters $S_{+}$ and $S_{-} . C_{S \pm}\left(\tau_{M}\right)$ corresponds to the probability distribution of the $S_{ \pm}\left(\tau_{M}\right)$, and is reflected in the statistical error bars $\Delta S_{ \pm}\left(\tau_{M}\right)$. The decay of $C_{S \pm}$ with $\tau_{M}$ is shown in Fig. 6. Fitting the data with exponential functions:

$$
C_{S \pm}=C_{S \pm}^{0} \exp \left(-\tau_{M} / \tau_{ \pm}\right), \quad \tau_{M}>0,
$$

gives $\tau_{+}=9.1 \pm 0.4 \mu \mathrm{s}$ and $\tau_{-}=8.1 \pm 0.3 \mu \mathrm{s}$, in good agreement with $\tau_{c}$. Note that $C_{S \pm}^{0}=2 C_{S \pm}\left(\tau_{M}=0\right)$, since $C_{S \pm}(\tau=0)$ is conditioned on two excitation in a same trial, while $C_{S \pm}(\tau>0)$ is conditioned on two excitations created in two different trials: the factor of two accounts for the two possible orders of excitations ('U' then 'D' or 'D' then 'U').

\section{APPENDIX E: CONDITIONAL CONTROL AND INCREASE IN GENERATION RATE}

As demonstrated in Ref. 25], the conditional control of remote memories enables a large enhancement of coincidence rates relative to the case where no logic is implemented. If the state prepared in one pair of ensembles
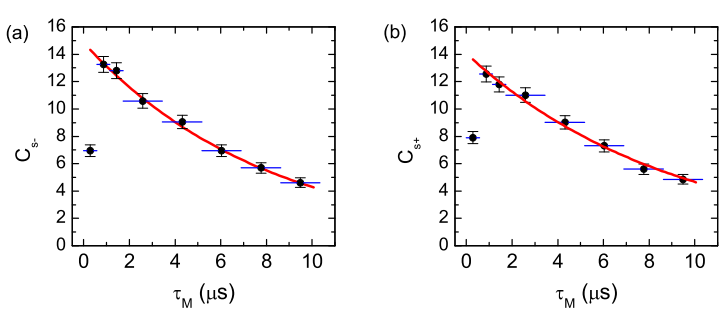

FIG. 6: The $\tau_{M}$ dependence of the total conditional count rates $C_{S \pm}$ in the measurement of (a) $S_{+}$and (b) $S_{-}$. The horizontal thick lines indicate the size of the memory bin. The error bars indicate statistical errors. The solid lines are fits using Eq. (D2).

is held up to $N$ trials, the rate for preparing both pairs is increased by a factor $(2 N+1)$ for very low excitation probability [25]. Figure 7(a) gives the probability $p_{11}$ of simultaneously preparing the two pairs. After 17 trials, an increase by a factor 34 is obtained experimentally, close to the expected value of 35 . The gain in the probability $p_{1122}$ of generating the effective entangled state is expected to be the same if the coherence time is long enough. However, our finite coherence time results in a smaller increase of the probability to detect field 2 coincidences. This increase is given in Fig. T(b), with a comparison to the ideal case of very long coherence time. A 19 -fold enhancement is finally obtained. Let us note that the different experimental rates can be obtained from these probabilities times the number of trials per second $\left(\sim 2.36 \times 10^{5} / \mathrm{s}\right)$.
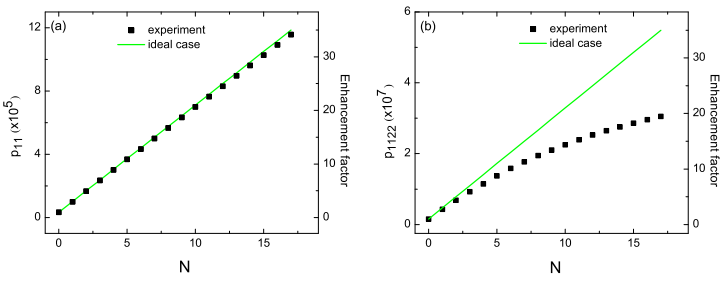

FIG. 7: Probabilities of coincidence detection as functions of the number of trials $N$ for which the first prepared pair holds the state. (a): measured probability $p_{11}$ of preparing the two pairs. (b): measured probability $p_{1122}$ of detecting field 2 coincidences. The green solid line corresponds to the ideal case of very long coherence time. Both panels give in addition to these probabilities the enhancement factor obtained relative to the case without conditional control. 


\section{APPENDIX F: CORRELATION FUNCTIONS $E\left(0^{\circ}, \theta_{R}\right), E\left(45^{\circ}, \theta_{R}\right)$ FOR THE IDEAL EFFECTIVE STATE}

In practice, various imperfections lead to deviations from the ideal effective state, Eq. (2) in the main text. We have developed a detailed model relevant to our experiment, but consider here only a generic form. Col- lective excitations are not shared with equal amplitudes between a pair of ensembles because of nonidealities in the writing and heralding processes. Likewise, the mapping of atomic states to states of field 2 by the read pulses is not ideal. Overall, these various imperfections lead to a state $\left|\psi_{2_{L U}, 2_{R U}, 2_{L D}, 2_{R D}}\right\rangle$ for field 2 given by (neglecting multi-photon processes):

$$
\begin{aligned}
\left|\psi_{2_{L U}, 2_{R U}, 2_{L D}, 2_{R D}}\right\rangle= & \left(\epsilon_{R U}\left|0_{2_{L U}}\right\rangle\left|1_{2_{R U}}\right\rangle \pm e^{i \eta_{U}} \epsilon_{L U}\left|1_{2_{L U}}\right\rangle\left|0_{2_{R U}}\right\rangle\right) \\
& \otimes\left(\epsilon_{R D}\left|0_{2_{L D}}\right\rangle\left|1_{2_{R D}}\right\rangle \pm e^{i \eta_{D}} \epsilon_{L D}\left|1_{2_{L D}}\right\rangle\left|0_{2_{R D}}\right\rangle\right) \\
= & \epsilon_{R U} \epsilon_{R D}\left|0_{2_{L U}}\right\rangle\left|0_{2_{L D}}\right\rangle\left|1_{2_{R U}}\right\rangle\left|1_{2_{R D}}\right\rangle \pm e^{i \eta_{U}} e^{i \eta_{D}} \epsilon_{L U} \epsilon_{L D}\left|1_{2_{L U}}\right\rangle\left|1_{2_{L D}}\right\rangle\left|0_{2_{R U}}\right\rangle\left|0_{2_{R D}}\right\rangle \\
& \pm e^{i \eta_{U}} \epsilon_{R D} \epsilon_{L U}\left|1_{2_{L U}}\right\rangle\left|0_{2_{L D}}\right\rangle\left|0_{2_{R U}}\right\rangle\left|1_{2_{R D}}\right\rangle \pm e^{i \eta_{D}} \epsilon_{R U} \epsilon_{L D}\left|0_{2_{L U}}\right\rangle\left|1_{2_{L D}}\right\rangle\left|1_{2_{R U}}\right\rangle\left|0_{2_{R D}}\right\rangle
\end{aligned}
$$

where $\epsilon_{X}$ is the probability amplitude that a photon is created in field $2_{X}$. The first and second terms in the expansion correspond to the cases that the two excitations are both retrieved at node "right" and "left", respectively. Thus the effective state that yields one detection event at node "left" and the other at node "right" consists of the last two terms. After the fields are combined by $\mathrm{PBS}_{L}$ and $\mathrm{PBS}_{R}$, we get the (unnormalized) effective state of fields $2_{L}$ and $2_{R}$

$$
\left|\psi_{2_{L}, 2_{R}}\right\rangle_{\text {eff }}=\alpha\left|H_{2_{L}} V_{2_{R}}\right\rangle \pm \beta\left|V_{2_{L}} H_{2_{R}}\right\rangle,
$$

where $\alpha \propto e^{i \eta_{D}} \epsilon_{R U} \epsilon_{L D}$ and $\beta \propto e^{i \eta_{U}} \epsilon_{R D} \epsilon_{L U}$.

From the effective state $\left|\psi_{2_{L}, 2_{R}}\right\rangle_{\text {eff }}$, we can derive the various coincidence probabilities $P_{i j}, i, j \in\{a, b, c, d\}$, where $\{a, b, c, d\}$ refers to the detectors $D_{2\{a, b, c, d\}}$ for field 2 in Fig. 1 of the main text. When $\theta_{L}$ is fixed at $0^{\circ}$, we find (assuming unity detection efficiency)

$$
\begin{aligned}
P_{a c} & =|\alpha|^{2} \sin ^{2} \theta_{R} \\
P_{b d} & =|\beta|^{2} \sin ^{2} \theta_{R} \\
P_{a d} & =|\alpha|^{2} \cos ^{2} \theta_{R} \\
P_{b c} & =|\beta|^{2} \cos ^{2} \theta_{R} \\
E\left(0^{\circ}, \theta_{R}\right) & \propto P_{a c}+P_{b d}-P_{a d}-P_{b c}=-\cos \left(2 \theta_{R}\right)
\end{aligned}
$$

irrespective of the \pm sign.

By contrast, when $\theta_{L}$ is fixed at $45^{\circ}$, we obtain

$$
\begin{aligned}
P_{a c} & =\frac{1}{4}\left[1 \pm 2|\alpha||\beta| \cos \phi \cos \left(90^{\circ}-2 \theta_{R}\right)\right. \\
& \left.+\left(|\beta|^{2}-|\alpha|^{2}\right) \sin \left(90^{\circ}-2 \theta_{R}\right)\right],
\end{aligned}
$$

where $\phi=\arg (\beta)-\arg (\alpha)$. Let $\alpha=\cos \varphi$, and $\beta=\sin \varphi$. Denoting $\delta=45^{\circ}-\theta_{R}$, we have

$$
\begin{aligned}
P_{a c} & =\frac{1}{4}[1 \pm|\sin 2 \varphi| \cos \phi \cos 2 \delta-\cos 2 \varphi \sin 2 \delta] \\
P_{b d} & =\frac{1}{4}[1 \pm|\sin 2 \varphi| \cos \phi \cos 2 \delta+\cos 2 \varphi \sin 2 \delta] \\
P_{a d} & =\frac{1}{4}[1 \mp|\sin 2 \varphi| \cos \phi \cos 2 \delta+\cos 2 \varphi \sin 2 \delta] \\
P_{b c} & =\frac{1}{4}[1 \mp|\sin 2 \varphi| \cos \phi \cos 2 \delta-\cos 2 \varphi \sin 2 \delta] \\
E\left(45^{\circ}, \theta_{R}\right) & \propto P_{a c}+P_{b d}-P_{a d}-P_{b c} \\
& = \pm|\sin 2 \varphi| \cos \phi \cos 2 \delta .
\end{aligned}
$$

From the expression for $E\left(45^{\circ}, \theta_{R}\right)$, we see that the deviation of $|\alpha|$ and $|\beta|$ from the balanced value, $1 / \sqrt{2}$, will lead to reduction in the visibility of $E\left(45^{\circ}, \theta_{R}\right)$ fringes and thus the magnitudes of the CHSH parameters $S_{( \pm)}$. We believe that such an imbalance is responsible for the results displayed in Fig. 3(b) for $E\left(45^{\circ}, \theta_{R}\right)$ and Fig. 4 for $S_{( \pm)}$at $\tau_{M}=0$ in the main text, with measurements underway to quantify this association.

Note that another combination of $P_{i j}$ 's given above results in

$$
\begin{aligned}
F\left(45^{\circ}, \theta_{R}\right) & \equiv-P_{a c}+P_{b d}+P_{a d}-P_{b c} \\
& =\cos 2 \varphi \sin 2 \delta .
\end{aligned}
$$

$F\left(45^{\circ}, \theta_{R}\right)$ allows us to determine $\varphi$ and thus the magnitude of the coefficients $\alpha$ and $\beta$, independent of $\phi$. Specifically, the visibility of the $F\left(45^{\circ}, \theta_{R}\right)$ fringes normalized to that of $E\left(0^{\circ}, \theta_{R}\right)$ fringes yields $\cos 2 \varphi$. 
[2] P. Zoller et al., Eur. Phys. J. D 36, 203 (2005).

[3] G. Giovannetti, S. Lloyd, L. Maccone, Science, 306, 1330 (2004).

[4] J.I. Cirac, P. Zoller, H.J. Kimble, H. Mabuchi, Phys. Rev. Lett. 78, 3221 (1997).

[5] L.-M. Duan, H.J. Kimble, Phys. Rev. Lett. 92, 127902 (2004).

[6] H.-J. Briegel, W. Dür, J.I. Cirac, P. Zoller, Phys. Rev. Lett. 81, 5932-5935 (1998).

[7] B.B. Blinov, D.L. Moehring, L.-M. Duan, C. Monroe, Nature 428, 153 (2004).

[8] J. Volz et al., Phys. Rev. Lett. 96, 030404 (2006).

[9] J. Beugnon et al., Nature 440, 779 (2006).

[10] A. D. Boozer, A. Boca, R. Miller, T. T. Northup and H. J. Kimble, Phys. Rev. Lett. (2007); available as quant-ph/0702248

[11] J.F. Sherson et al., Nature 443, 557 (2006).

[12] L.-M. Duan, M. Lukin, J.I. Cirac, P. Zoller, Nature 414, 413 (2001).

[13] A. Kuzmich et al., Nature 423, 731 (2003).

[14] C.H. van der Wal et al., Science 301, 196 (2003).

[15] V. Balić, D.A. Braje, P. Kolchin, G.Y. Yin, S.E. Harris, Phys. Rev. Lett. 94, 183601 (2005).

[16] C.W. Chou, S.V. Polyakov, A. Kuzmich, H.J. Kimble, Phys. Rev. Lett. 92, 213601 (2004).

[17] M.D. Eisaman et al., Nature 438, 837 (2005).

[18] T. Chanelière et al., Nature 438, 833 (2005).

[19] J. Laurat et al., Opt. Express 14, 6912 (2006).

[20] J.K. Thompson, J. Simon, H. Loh, V. Vuletić, Science 313, 74 (2006).

[21] C.W. Chou et al., Nature 438, 828 (2005).

[22] H. de Riedmatten et al., Phys. Rev. Lett. 97, 113603 (2006).

[23] D.N. Matsukevich et al., Phys. Rev. Lett. 97, 013601 (2006).

[24] S. Chen et al., Phys. Rev. Lett. 97, 173004 (2006).

[25] D. Felinto et al., Nature Physics 2, 844 (2006); advance online publication, (doi:10.1038/nphys450).

[26] A. Ekert, Phys. Rev. Lett. 67, 661 (1991).

[27] C.W. Chou, PhD Thesis, Chapter 8, California Institute of Technology, May 2006, http://www.its.caltech.edu qoptics/thesis.html

[28] L. Jiang, J. M. Taylor, M. D. Lukin, quant-ph/0609236

[29] Z. B. Chen, B. Zhao, J. Schmiedmayer, J.-W. Pan, quant-ph/0609151

[30] D. Felinto, C.W. Chou, H. de Riedmatten, S.V. Polyakov, H.J. Kimble, Phys. Rev. A 72, 053809 (2005).

[31] J.F. Clauser, A. Shimony, Rep. Prog. Phys. 41, 1881 (1978).

[32] C.A. Fuchs, N. Gisin, R.B. Griffiths, C.S. Niu, A. Peres, Phys. Rev. A 56, 1163 (1997).

[33] I. Marcikic et al., Phys. Rev. Lett. 93, 180502 (2004).

[34] We gratefully acknowledge critical discussions with S. J. van Enk. This research is supported by the Disruptive Technologies Office (DTO) and by the National Science Foundation. J.L. acknowledges financial support from the European Union (Marie Curie fellowship). H.D. acknowledges support as Fellow of the Center for the Physics of Information at Caltech. 